\title{
Les contacts linguistiques bulgaro-roumains
}

\section{Georgi Armianov}

\section{OpenEdition}

\section{Journals}

Édition électronique

URL : https://journals.openedition.org/cher/7895

DOI : $10.4000 /$ cher.7895

ISSN : 2803-5992

\section{Éditeur}

Presses universitaires de Strasbourg

\section{Édition imprimée}

Date de publication : 1 décembre 2009

Pagination : 29-38

ISBN : 978-2-35410-006-3

ISSN : 1968-035X

Référence électronique

Georgi Armianov, "Les contacts linguistiques bulgaro-roumains », reCHERches [En ligne], 3 | 2009, mis en ligne le 15 décembre 2021, consulté le 17 décembre 2021. URL : http://journals.openedition.org/ cher/7895; DOI : https://doi.org/10.4000/cher.7895

\section{cc) (i) (-)}

Ce(tte) œuvre est mise à disposition selon les termes de la Licence Creative Commons Attribution Pas d'Utilisation Commerciale - Partage dans les Mêmes Conditions 4.0 International. 


\title{
Les contacts linguistiques bulgaro-roumains
}

\author{
Georgi Armianov \\ INALCO
}

Tes relations linguistiques bulgaro-roumaines ont été l'objet de plusieurs $\mathcal{L}$ recherches et analyses historiques, dialectales, lexicales, grammaticales. On observe également une augmentation constante du nombre des dictionnaires phraséologiques, thématiques et bilingues et des monographies publiés. Notre objectif ici sera de faire une synthèse des moments les plus importants dans ces contacts, de montrer les influences à travers les siècles et de tracer, autant que possible, une perspective. Dans notre travail, nous nous limiterons principalement à l'influence du bulgare sur la langue roumaine même si nous produisons des exemples et des réflexions dans l'autre direction.

Du point de vue historique, nous pouvons affirmer avec certitude que les contacts linguistiques et culturels entre ces deux peuples ont commencé dès l'Antiquité. Les deux anciens peuples voisins des Thraces et des Daces ${ }^{1}$, dont les Bulgares et les Roumains sont dans une certaine mesure les descendants contemporains, avaient une origine commune. Selon les sources historiques et toponymiques assez rares, leurs langues aussi étaient très proches. Pourtant, la proximité des Thraces des anciens territoires grecs ainsi que l'existence du Danube comme barrière politique et culturelle naturelle, ont séparé leurs destins. Du fait de l'absence d'écriture propre aux Thraces, ils ont été progressivement hellénisés, tandis que ce processus est resté beaucoup plus faible, marginal pour leurs cousins, les Daces. D'un autre côté, ce voisinage avec les grandes puissances de l'Antiquité européenne a

1 Les Daces sont connus également sous les noms de Thraces, Tyrgètes et Gètes.

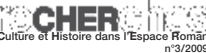


permis une meilleure description de la vie et des mœurs des Thraces. C'est grâce aux annalistes et historiens grecs que nous possédons aujourd'hui une connaissance des tribus, des rois, des centres politiques et culturels des anciens États thraces. Grâce à eux, nous acquérons une image, même parfois limitée, de la toponymie et de l'hydronymie des terres entre le Danube, l'ancien Istros (en grec "I $\sigma \tau \rho \circ$ ), et le Péloponnèse.

Après cette étape initiale de colonisation, l'histoire des Daces et des Thraces se rapproche considérablement avec l'occupation et l'incorporation aux $\mathrm{I}^{\text {er }}$ et $\mathrm{II}^{\mathrm{e}}$ siècle des territoires des Balkans ${ }^{2}$ à l'Empire Romain. Or, la romanisation des populations thrace et dace suit des voies différentes, surtout linguistiques. Les Thraces, déjà hellénisés, n'acceptent pas pleinement le latin comme langue commune, car le grec, qui a remplacé les dialectes thraces dans les contacts quotidiens, reste toujours une des langues officielles de l'Empire Romain. Au contraire, l'ancienne langue des Daces est progressivement remplacée par le latin qui donne au fil du temps naissance à l'actuelle langue roumaine.

Quelques siècles plus tard, vers la fin du $v^{e}$ et le début du vi $I^{e}$ siècle, les Balkans sont envahis par les Slaves, qui se mélangent à la population locale hellénisée et romanisée et imposent graduellement leur langue, surtout au sud du Danube. Puis, au milieu du viI siècle, arrivent les Protobulgares, un peuple originaire de l'Altaï, qui s'installent initialement sur le territoire situé au nord du Danube, dans la Dobroudža du Nord, près de l'estuaire, dans un

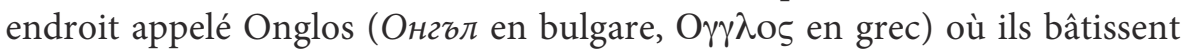
leurs premières forteresses et habitations ${ }^{3}$. Progressivement ils traversent le fleuve et occupent les terres jusqu'à la chaîne du Balkan. Là, s'unissant aux tribus slaves et aux descendants des anciens Thraces, ils fondent le premier

2 Le terme Balkans est proposé pour la première fois et utilisé dans des recherches scientifiques par le géographe allemand Johann August Zeune en 1808 (voir, Glenny 2000 , p. XXII). Pour les objectifs de notre travail, nous l'utilisons ici dans un sens plus large et plus libre, en désignant ainsi les terres de l'Europe du sud-est de l'époque antique et médiévale.

3 La question de l'endroit exact de cette forteresse fait toujours l'objet de débats. La plupart des spécialistes considèrent qu'elle se trouvait près du delta du Danube, dans les terres de la Roumanie, de l'Ukraine et de la Moldavie contemporaines. Au début du xx siècle, près du village Nikolicel et de la ville d'Issacea en Roumanie, les archéologues ont découvert les ruines d'une forteresse dont le plan est presque identique à celui de la forteresse de Pliska, la première capitale bulgare. Or, cette citadelle fortifiée du Danube avait une superficie de $48 \mathrm{~km}^{2}$, deux fois plus grande que Pliska. Les archéologues roumains pensent que ce sont très probablement les ruines de l'ancienne citadelle du khan bulgare Asparukh, avant qu'il traverse le fleuve pour fonder le Royaume bulgare sur les Balkans, c'est-à-dire probablement de l'ancien Onglos. 
royaume bulgare auquel ils donnent leur nom ainsi que la structure et l'organisation de l'État, alors que les slaves laissent leur langue. De surcroît, il est fort intéressant de noter que la région située au nord du Danube demeure pour plusieurs siècles, de 681 jusqu'à 1241, partie intégrante du premier royaume bulgare.

Cette situation explique l'influence naturelle qui va s'exercer sur le développement du peuple qui habite à cette époque les territoires de la Roumanie actuelle. La population garde sa langue maternelle daco-latine (ou daco-roumaine) pour ses contacts ordinaires, quotidiens, alors que le bulgare devient la langue de l'administration du pays et de l'aristocratie.

De cette époque date exactement la première mention de l'ethnonyme Valaque $^{4}$ : nous la trouvons dans une chronique de l'historien byzantin Kedrenos de 976 qui raconte l'assassinat par des Valaques de comitopoul 5 David, frère du futur roi bulgare Samouil. Puis, le terme a été utilisé officiellement par le pape Innocent III en 1205 dans sa correspondance avec le roi bulgare Kalojan (1197-1207) à qui il a attribué le titre de rex Bulgarorum et Blachorum "roi des Bulgares et des Valaques» (Constantinescu, 1970: 98).

En 1330, la première principauté valaque a été fondée et le bulgare médiéval commence à jouer le rôle de langue officielle de l'État et de l'Église orthodoxe valaque. La présence du bulgare depuis quelques siècles et sa nouvelle fonction laissent également une empreinte sur la langue de la population ordinaire qui adopte un grand nombre de mots d'origine bulgare. C'est l'historien allemand Schlözers qui, au XVIII ${ }^{\mathrm{e}}$ siècle, mentionne pour la première fois l'existence et l'influence du bulgare sur la langue roumaine. Dans son ouvrage "Allgemeine Nordische Geschichte", il dit: "Beaucoup plus de la moitié de leurs mots (des Roumains) ainsi que leur grammaire sont empruntés d'une langue inconnue jusqu'à présent, probablement le vieux bulgare» (Schlözers, 1771: 253).

$\mathrm{Au}$ début du $\mathrm{XIx}^{\mathrm{e}}$ siècle, le problème de l'évolution de la langue roumaine a été étudié par les slavistes slovènes Bartoloměj Kopitar et Fran (Frantz) Miklošič qui soulignent le rôle important du vieux bulgare dans

4 Selon certains historiens et linguistes, le terme «valaque» provient de l'exonyme Walch par lequel les Goths désignaient au début les Volsques, peuple celtique avec lequel ils étaient en contact dans leurs territoires méridionaux et puis tous les peuples celtoromans: les Wallons en Belgique ou les Gallois (les Welsh) sur les îles britanniques. Jusqu'à aujourd'hui, les Bulgares appellent tous les peuples romains des Balkans avec le nom commun «vlakh», pl. «vlasi».

5 Du grec Kó $\rceil_{\uparrow}$ 'comte, gouverneur régional'. 
ce processus. Les mêmes questions sont aussi analysées par le folkloriste et linguiste roumain Ovid Densuşianu dans son Histoire de la langue roumaine où il atteste que la plupart des éléments slaves ou slavo-bulgares ont pénétré dans la langue roumaine déjà au cours du vir ${ }^{\mathrm{e}}$ siècle (Densusianu, 1938).

Évidement, à l'époque ancienne, et même au $\mathrm{Xv}^{\mathrm{e}}$ siècle, l'influence du bulgare est restée très forte. Dans un article sur la langue des Décrets valaques de cette période (appelés également Chartes valaques), Ljubomir Milétitch affirme:

À l'époque des décrets les plus anciens [...], le roumain prédomine déjà dans la vie publique et privée du pays. [...] D’un autre côté, cependant, au moment où furent rédigés les décrets de Mircea le Grand et de ses premiers successeurs (jusque vers les années 1410-1450 au plus tard), il y avait toujours une partie de la population, aussi bien parmi le petit peuple que parmi les boyards, qui parlait le bulgare (Милетич 1908: 24).

En 1929, dans Geschichte der bulgarischen Sprache, publié premièrement en allemand à Berlin et à Leipzig, Stefan Mladenov consacre deux chapitres entiers à la question des contacts entre le bulgare (et le vieux bulgare) et le roumain où il analyse plusieurs problèmes de la phonétique et la morphologie: la réduction des voyelles bulgares, la forme du futur, l'article postposé, etc. ${ }^{6}$ Il critique également certaines théories de Benjo Tsonev sur le même sujet présentées dans l'étude Contacts linguistiques entre Bulgares et Roumains (Tsonev/ Цонев, 1921).

Mladenov souligne également que la plus grande partie des éléments lexicaux slaves dans la langue roumaine se distingue par leurs particularités grammaticales bulgares, comme les combinaisons $\check{s} t$ et $\check{z} d$ qui remplacent les anciens ${ }^{*} t j$ et ${ }^{*} d j$ indo-européenne, par exemple, sfeştanie 'bénédiction de l'eau' du vieux bulgare св Аштение, moştean 'héritier' du vieux bulgare моштьнъ 'capable, fort', odăjdii 'vêtements' de одежда, etc., ou les correspondances roumaines des anciens jers bulgares $b$ et $b$ dans des mots comme văzduh 'air' dе въздоухъ, păstrăv 'truite' de пьстръ 'bigarré', temniță 'prison' de тьмьница, dobitoc 'bétail' de vieux bulgare добытъкъ, etc. (Mladenov/ Младенов, 1979: 69-70).

Quelques décennies plus tard, en 1948, dans ses Discussions dans le domaine de la dialectologie historique bulgare: la langue des décrets valaques des $X I V^{e}-X V^{e}$ siècles, le linguiste russe Samuel Bernstein analyse le

6 Le livre Geschichte der bulgarischen Sprache de Stefan Mladenov a été traduit en bulgare par Prof. Ivan Duridanov et publié en 1979. Les citations ici sont données selon l'édition bulgare. 
développement de la langue populaire bulgare dans les territoires valaques et son influence sur la langue de l'administration et de l'État valaque de l'époque de Mirtcha I (1386) jusqu'à Radu IV (1508). Il donne plusieurs exemples de mots et même de phrases entières non seulement de la correspondance officielle de l'État, mais de la langue populaire, mentionnés dans ces documents, tels que: больр 'boyard', падина 'vallon', полица 'étagère', невеаста 'jeune mariée', nраг 'seuil', cumy 'tamis' (avec une réduction pleine de la voyelle $\mathrm{O}$ ), бамза 'père', воденица 'moulin à eau', голям 'grand', грижа 'sоuсi', девойка 'fille', детияа (деиа) 'enfants', да доидеm 'venir', завъриих 'j'ai fini', защо 'pourquoi', зидар 'maçon', нuе 'nous', нищо 'rien', оти (защо, защото) 'pourquoi; parce que', cват 'gendre', сирене 'fromage', тази 'cette', тези 'сеих', тъкмеж 'préparation', устие 'estuaire', хубав 'beau', мура (мурей) 'beau-frère', mais aussi, ce qui est très intéressant, des mots comme кожух / кожуси 'manteau de fourrure', сирак/ сираци 'orphelins', сиромах/ сиромаси 'pauvres', юнак/ юнаци 'héros' qui montrent la deuxième palatalisation des consonnes $\Gamma, \mathrm{K}, \mathrm{X}$ en 3 , Ц, C, caractéristique de la langue bulgare de l'époque médiévale jusqu'à nos jours (Bernstein / Бернщейн, 1948: 204 ).

Nous découvrons également des influences dans la grammaire de ces documents, où on trouve des caractéristiques phonétiques et morphologiques bulgares dans des mots d'origine slave, par exemple: $\sigma \mathcal{M}(\partial \mathrm{m})$ et $\boldsymbol{\sigma H}(\partial \mathrm{n})$ à la place de la voyelle nasale $\pi$ : $з б \mathcal{M} б u$ 'dents', $к р б н г$ 'cercle', дъмбрава 'forêt', мънка 'douleur, mal' et in à la place de la voyelle nasale $\mathrm{A}$ - оглінда 'examiner tour à tour, étudier, etc.

Dans la morphologie, les particularités sont plus nombreuses. Le futur est construit avec la particule щъ (де хотяъти - хощх 'vouloir'), liée à l'infinitif, ce qui est une construction spécifique du vieux bulgare et du bulgare médiéval, parexemple: щет учинит 'fera' (dans le Décret de Mircea), a miе му щ, пт платит 'et ils lui payeront' (Décret de Mihail), не щете имати страх от турии 'il ne faut pas avoir peur des turcs (Décret de Basarab), азь не щъљ оставит тіази работа 'je ne laisserais pas cette histoire' (Décret de Vladislav), etc. (Bernstein/ Бернщейн, 1948: 204-206).

La forme de l'aoriste est présente aussi régulièrement, et il s'agit notamment de ce qu'on appelle «le nouvel aoriste sigmatique bulgare» du type несохъ, par exemple: писах въ столни град 'j'écrivis à la ville royale' (Décret de Radu IV), како се отметняхме от господина краль и дадохме се турием 'comment nous avons renié notre maitre le roi et nous nous 
sommes rendu aux Turcs' (Décret d'Alexandre) (Bernstein / Бернщейн, 1948: 207-209).

$\mathrm{La}$ première personne $\mathrm{du}$ singulier $\mathrm{du}$ pronom personnel dans les documents cités est le plus souvent exprimée par la forme bulgare A3 ( $a 3$, aзr et ázb), qui n'est pas typique des autres langues slaves. Les pronoms des première et deuxième personnes du pluriel - нue et вue - reflètent également des particularités bulgares: "ніе служимо господину кралю» 'nous servons notre maître le roi', «а вие го целуваите» 'et vous l'embrassez'. De surcroît, on rencontre les formes courtes des pronoms possessifs comme: редом със сина му 'avec son fils', понеже покупише от баша им 'parce qu'ils ont acheté de leur père». Ici, dans le deuxième exemple, nous trouvons également une forme de l'ancien aoriste asigmatique (покупише 'ils ont acheté) qui existe encore aujourd'hui dans certains dialectes régionaux bulgares, notamment ceux de l'ouest.

Dans les décrets les plus anciens, les pronoms interrogatifs et relatifs sont d'habitude $\kappa m o /$ чmo ou $x m o$, mais depuis la première moitié du $\mathrm{xv}^{\mathrm{e}}$ siècle on trouve aussi les formes bulgares contemporaines кой et който, par exemple: конник кои минет мимо Турч, и които ходит на купля 'lе chevalier qui passe près de (la ville de) Tourtcha et qui va au marché. De la même façon, l'ancien pronom négatif нuxmo - par exemple, “...u om турии нихто да се не боит"' ...et que personne n'a peur des Turcs' - a été probablement remplacé par la nouvelle forme никой.

Naturellement, dans les documents de cette époque nous trouvons beaucoup de noms propres qui montrent une origine slave, surtout bulgare, et qui attestent une présence significative de personnes d'origine bulgare dans les principautés valaques, tels que: Stancho, Stajko, Gergin, Dragomir, qui furent des boyards dans les cours des princes valaques, ou encore le toponyme Tărgovište, la capitale du prince Vlad Ier Dracula, (un nom dans lequel nous découvrons la racine ${ }^{\star} T r g o v$ ' 'faire du commerce' et le Щ typiquement bulgare qui provient $\mathrm{du}{ }^{*} \mathrm{tj}$ indo-européen), ou la ville de Dumbovitsa avec l'ancienne voyelle nasale $\pi$, Dobrotitsi 'la région de la Dobroudža' dont l'origine est l'adjectif добръ 'bon', Bistritsa de бистръ 'claire, transparent', Racovița de paк 'écrevisse, crabe', Vranča de врана 'corneille', Vălcani de вълк 'loup' et beaucoup d'autres.

L'occupation de la Bulgarie par les Turcs ottomans en 1393-1396 met fin aux relations officielles entre la Principauté valaque et le Royaume bulgare. Beaucoup de Bulgares trouvent refuge chez leurs voisins en Valachie et cette émigration devient très forte au début $\mathrm{du} \mathrm{XvII}^{\mathrm{e}}$ siècle, après les rebellions 
manquées des catholiques bulgares. Les habitants des villes et des villages entiers quittent leur pays natal et s'installent dans la région du Banat et en Valachie où ils créent plusieurs grandes colonies bulgares qui gardent leur langue et leurs traditions pendant des siècles. Au début des années 1950, le dialectologue bulgare St. Stoikov fait de larges recherches sur les dialectes bulgares en Roumanie et constate des choses très intéressantes (Stoikov/ Стойков, 1957; Стойков, 1968 ).

Il trouve approximativement 17500 Bulgares installés majoritairement dans les villages de Vinga, Bešenov, Breškya, Denta et Telepa (Colonia Bulgară) en Banat. Une partie d'entre eux sont des descendants des Bulgares catholiques et orthodoxes qui ont quitté la Bulgarie après l'échec de la révolte de Čiprovtsi en 1688. Ils se sont réunis en Valachie avec beaucoup d'autres Bulgares catholiques de la région de Svištov et de Nikopol qui, étant plus nombreux, ont progressivement imposé leur dialecte bulgare oriental.

À cette époque ancienne, la région du Banat faisait partie de l'Empire austro-hongrois et les autorités interdisaient l'utilisation libre du bulgare et la publication de livres bulgares. Par conséquent, la grammaire de ce dialecte oral bulgare du XviI ${ }^{e}$ siècle reste figée alors que le vocabulaire est dilué dans un grand nombre de mots d'origine croate (car au début leurs prêtres étaient souvent d'origine croate), hongroise, allemande et roumaine. En même temps, dans la communication quotidienne de la population roumaine, on voit entrer des mots liés à la vie quotidienne et aux traditions bulgares, comme: lopatica 'pelle', grebla 'râteau', obor 'étable', evța/ ovța 'brebis', ovas 'avoine', rîjnița 'seigle', pleava 'glume', boaba 'haricot', morcov 'carotte', svecla 'betterave', lubenița 'pastèque', prost 'stupide', prostac 'nigaud', voire l'exclamation «Ox, Боже, Боже!» 'Oh, mon Dieu!' Dans son livre «Une île de latinité», l'écrivain roumain Mircea Goga présente plusieurs mots, parfois avec certaines différences sémantiques, dont l'origine est bulgare, par exemple: ochi 'yeux', obraz 'la joue', buza 'lèvre', glezna 'cheville', ceaşcă 'tasse', miros/miris 'odorat', pipăit 'toucher' et beaucoup d'autres (Goga, 2004: 87-95).

Vers la fin du $\mathrm{xVIII}^{\mathrm{e}}$ siècle la Valachie et les territoires roumains, qui ont gardé une plus grande autonomie au sein de l'Empire ottoman, deviennent un endroit sûr pour les Bulgares persécutés et pour la nouvelle intelligentsia bulgare. Bucarest et Djurdju (Гюргево, en bulgare) accueillent non seulement des émigrés et des ouvriers saisonniers, mais aussi des enseignants, marchands, écrivains et chercheurs. C'est en Roumanie que l'une des premières figures de la Renaissance bulgare, le prêtre Sofronij de 
Vratsa, trouve refuge et publie certains de ces ouvrages. En 1824, toujours en Roumanie, le docteur Petăr Beron, médecin et homme de lettres qui parlait neuf langues, écrit et publie le premier manuel laïc moderne bulgare, L'abécédaire avec divers enseignements (connu aussi sous le titre L'abécédaire au poisson), et plus de trente livres, deux dictionnaires et un atlas. À Bucarest, le docteur Nikola Savov-Picollo prépare ses ouvrages de médecine. Les célèbres révolutionnaires Georgi Rakovski, Ljuben Karavelov et Christo Botev y travaillent, font leurs plans pour la libération de la Bulgarie et publient leurs journaux.

Les contacts de la nouvelle bourgeoisie commerçante et de l'intelligentsia bulgare avec la culture roumaine et, à travers elle, avec les langues et cultures européennes contribuent naturellement à la naissance et au développement de la langue moderne bulgare. Par le biais du roumain, le bulgare s'enrichit de mots appartenant au lexique international comme masa 'table', telegraf 'télégraphe', poshta 'poste', militsiya 'milice, police', ou des noms comme Frantsiya 'France', Pariž 'Paris'. On trouve des mots de la langue courante, tels que bordej 'bordel', botuš 'botte', guša 'gorge', dalavera de roumain daravera 'affaire commerciale', carnače de roumain cirnaț 'saucisse', lanets 'chaînette', mamaliga 'polenta', cincarin 'aroumain', mais également le mot kachakaval 'fromage de brebis ou de vache du type gouda' qui est d'origine italienne et qui est considéré par la plupart des Bulgares comme un mot certainement bulgare.

Il est intéressant de souligner ici le mot mokanin 'berger', dont l'utilisation était confinée au Nord-est de la Bulgarie, mais qui est devenu très populaire avec le récit $L$ 'hirondelle blanche du célèbre écrivain bulgare Jordan Jovkov, qui a travaillé à Bucarest en tant que secrétaire à l'ambassade bulgare.

Après la Seconde Guerre mondiale, plusieurs écoles bulgares ont été créées en Roumanie dans lesquelles l'enseignement se fait aujourd'hui en roumain et en bulgare. Il y a aussi quelques journaux, comme "Náşa glás. Organ na balgarskotu družstvu ud Banáta» par exemple, écrits en bulgare mais en caractères latins. Récemment la chaîne de la télévision bulgare Kanal 1 a montré le film 3924 kilomètres à travers la Roumanie qui raconte l'histoire de la population d'origine bulgare en Roumanie. De surcroît, le Centre régional de la télévision bulgare à Rousse a présenté un autre film Des racines ailleurs en quatre épisodes consacré à la vie et l'histoire des Bulgares vivant dans les régions du Banat (les villages de Star Bešenov/ Dudešti Vechi, Breskia et Vinga) et de Bessarabie (aujourd'hui en Moldavie et en Ukraine). 
Malheureusement, il y a très peu de recherches récentes sur la langue de la population d'origine bulgare en Roumanie et, en général, elles sont incluses dans d'autres monographies (Бончева / Лучев 2000 : 65-74); Лучев 2002; Нягулов 1999).

Les contacts directs entre les deux langues sont actuellement limités par le fait qu'elles sont déjà devenues des langues standard développées à la grammaire et au vocabulaire sont stables et qu'elles sont directement et largement ouvertes sur les autres langues du monde.

\section{Bibliographie}

Constantinescu, Mihail, 2000, Histoire de la Roumanie de origines à nos jours, Paris, Ed. Horvath.

Densuşianu, Ovide, 1938, Histoire de la langue roumaine, Paris, Leroux.

Glenny, Misha, 2000, The Balkans 1804-1999, London, Granta Books.

Goga, Mircea, 2004, Une île de latinité (Culture, civilisation, langue et littérature roumaine), Paris, Presses de l'Université Paris-Sorbonne.

Schlözers, August, 1771, Allgemeine Nordische Geschichte, Halle.

Бернштейн, Самуел, 1948, Язык валашских грамот XIV-XV веков [La langue des décrets valaques de XIV-Xv siècles], in Разыскания в области болгарской исторической диалектологии [Discussions dans le domaine de la dialectologie historique bulgare], Moscou / Léningrad, t. 1.

Бончева, Цветана еt Детелин Лучев, 2000, “Съвременни аспекти на идентичността при българските католици”, in Ирена Бокова, Локални общности, Том 1, Културна идентичност - Традиции - Регионална принадлежност, София, Издателство „Яр”.

Лучев, Детелин, 2002, “Аспекти на проблема за идентичността на „банатските българи в България”, in V. Tepavicharov, Ethnologia Academica 3 - Conference: "The Balkans and the Culture of the Balkan Peoples", Etropole 2000, Sofia.

Милетич, Любомир, 1908, Към грамотите от брашовската сбирка [Au sujet des décrets de la collection de Braşov], in СбНУ, 1908, София, т. XXV.

Младенов, Стефан, 1979, История на българския език, София, Издателство на Българската академия на науките.

Нягулов, Благовест, 1999, Банатските българи. Историята на една малиинствена общност във времето на наиионалните държави, София, Издателство «Парадигма». 
Стойков, Стойко, 1957, Банатският говор, София, Издателство на Българската академия на науките.

Стойков, Стойко, 1968, Лексиката на банатския говор, София, Издателство на Българската академия на науките.

Цонев, Беньо, 1921, “Езикови взаимности между българи и румъни”, Годишник на Софийския университет, Историко-филологически факултет, София, т. XV-XVI, 1921, p. 1-158. 